\title{
NEW OBSERVATIONAL ASPECTS
}

\author{
ODDBJØRN ENGVOLD \\ Institute of Theoretical Astrophysics \\ University of Oslo \\ P.O.Box 1029, Blindern \\ N-0315 Oslo 3, Norway
}

\begin{abstract}
The requirements and conditions for high resolution imaging and polarimetry of the Sun are reviewed. Various methods and techniques are discussed for image stabilization and sharpening in solar observations. The new solar facilities in the Canary Islands in particular are frequently reaching diffraction limited resolution and yield new insight in the structure and dynamics of the solar atmosphere. Future ground based telescopes like THEMIS and LEST, as well as planned solar missions in space will trigger a next advance in solar physics.
\end{abstract}

\section{Introduction}

The future of solar physics lies in understanding the basic astrophysical processes that can be observed on the Sun. This includes understanding the magnetohydrodynamical processes that occur, and an important, even crucial, tool for understanding these processes is polarimetry, combined with spectroscopy, of small spatial domains over extended periods of time.

It is increasingly apparent to solar astronomers that further progress in understanding the physics of the solar atmosphere relies critically on observations of sub-arcsecond structures. Many fundamental physical processes in the Sun's atmosphere obviously occur at sub-arcsecond scales. Recent analysis of the C IV emission of the solar transition region suggests that emitting structures may be $\leq 10 \mathrm{~km}$ (Dere 1987). The sizes of the smallest structures in the Sun's atmosphere might possibly be the photon mean free path at $\tau_{c} \sim 1$ in the photosphere, which is about $50 \mathrm{~km}$ in the visible, and $\leq 50 \mathrm{~km}$ at the opacity minimum around $1.6 \mu \mathrm{m}$. In the presence of magnetic fields in the solar atmosphere the oscillatory modes of small flux ropes (Ryutova; this proceedings) may constitute spatial scales that are only a fraction of the assumed flux rope diameters $(\leq 300 \mathrm{~km})$. An even smaller and more relevant scale would seem to be the thickness of boundary layers which are matching local gyro radii and are therefore in the $m$ and $\mathrm{cm}$ ranges. The characteristic time scales of the fine structure elements can be tied to the sizes and motions of the structures. Assuming a conservative value for the element size; $d \sim 70 \mathrm{~km}$, and characteristic 
velocity $\leq 2 \mathrm{~km} \mathrm{~s}^{-1}$ and $\leq 10 \mathrm{~km} \mathrm{~s}^{-1}$ in respectively the photosphere and chromosphere, we find corresponding time scales $d / v \sim \frac{1}{2} \min$ and $\leq 5 s$.

One will clearly not be able to resolve the smallest of such solar structures in the foreseeable future, but every improvement in spatial and temporal resolution is likely to yield new insight and understanding of the physics of the Sun. Exciting and highly worth while projects for the near future are such as the ground based THEMIS and LEST, and possibly the space borne OSL, and these will presumably offer resolution in the range 0.15 - 0.05 arcsec, which is better than the best telescopes today.

\section{Observational Requirements}

\subsection{General Requirements}

The requirements for a modern optical solar telescope are: (1) high spatial resolution, (2) high system through-put, and generally also (3) low instrumental polarization. The near infrared wavelength region allows observations to deeper layers of the photosphere and (4) near IR capability is desirable. In addition, the observations done with todays solar telescopes, from ground as well as from space, present a significant challenge in (5) data management capabilities which includes (i) acquistion, (ii) transfer, (iii) calibration, (iv) reduction, (v) analyzing, and (vi) archiving. The problem is not unique to large telescopes only. Given combinations of high spatial, spectral and temporal resolution over even a field of view of few arcsec is generally a major data handling task for any good solar telescope.

The effective spatial resolution attainable with a given telescope system is a function of the optical quality of the system itself and the seeing. The main contribution to the seeing is from the atmosphere, but a non-negligible share comes from the local telescope environment. The careful choice of site is equally important to the optical and mechanical quality of the telescope system. Also a good site may easily be spoiled if the telescope and tower structure generate strong air turbulence.

A solar site is considered to be superb if it offers seeing of $0.1-0.3$ arcsec a fraction of the time. This fraction can be notably enhanced with the aid of the real-time techniques active and adaptive optics (Beckers 1989c). Furthermore, solar interferometry techniques can be applied after the fact to recover the high spatial frequency signals down to the diffraction limit of the telescope (von der Lühe and Zirker 1988). The "Knox-Thompson" scheme (Knox and Thompson 1974) shows promise and has been demonstrated by Stachnik et al. (1983) and von der Lühe (1988a). The future prospects in solar interferometry is discussed by Rozelot (this proceedings).

Remote operations/observing of stellar telescopes are presently developed and tested for the purpose of enhancing the efficiency in use and lower the cost (Martin and Hartley 1985; Raffi 1988). Remote observing is planned as an option in LEST (Engvold et al. 1985).

In the following we shall address some of the points above.

\subsection{Spatial Resolution}

In a perfect imaging system all energy of a point image is concentrated in an Airy disc corresponding to the diffraction of the circular telescope aperture. The angle corresponding 
to the radius of the central Airy disc is usually considered as the telescope resolution. The modulation transfer function corresponding to this resolution is 0.0894 . This means that, say, a true $15 \%$ contrast of solar granulation is reduced to less than $1 \%$ in the image produced by a $\sim 10 \mathrm{~cm}$ aperture telescope that just "resolves" the granular structure. The effects from telescope aberrations and seeing add to this and serve to reduce the spatial resolution further.

The quality of an imaging optical system is quantified by comparison to the theoretical diffraction image. The ratio of central intensity of the point image theoretical central intensity is the Strehl Ratio (SR). When the rms deviations from the perfect surface is expressed in terms of phase error $\Delta \Phi$ the resulting Strehl Ratio becomes (see Dunn 1987):

$$
S R=\left(1-\Delta \Phi^{2}\right)
$$

A common convention is to consider a system diffraction limited when $S R$ is greater than or equal to 0.8 , which implies $\Delta \Phi=0.447$ or the equivalent to $\lambda / 14$. An $r m s$ phase error corresponding to $\lambda / 20(\Delta \Phi=0.314)$ yields $S R=0.90$. The phase errors due to atmospheric seeing will affect the $S R$ in a similar way. A partially correted phase variation of the wavefront due to seeing (see Sections 4.2 and 4.3) will result in a point spread function that consists of a sharp diffraction disc superposed on a residual halo-like image with the shape of the seeing disc (Roddier and Roddier 1986). The resulting image in this case will be a composite of a diffraction limited and a blurred component.

\subsection{The Light Flux and Time Resolution}

The number of available photons in the detector plane are generally abundant in solar observations at medium resolution (1-2 arcsec) and over medium to broad spectral bands $(\Delta \lambda>1 \AA)$. On the other hand, in the case of high resolution, Stokes polarimetry one needs a large aperture solar telescope with high throughput in order to attain the required signal to noise ratio. Besides increasing the collecting aperture area of the telescope one also have to use good detectors. Highly efficient charge-coupleddevices ("CCD") detector arrays offer quantum efficiencies up to $50 \%$ and more in the visible and near IR, compared to 2 - $25 \%$ for photomultipliers, and $\sim 1 \%$ for photographic emulsions.

The number of photons per unit time at the detector plane of a telescope with aperture diameter $D$ and focal length $f$ can be expressed by:

$$
N=0.5 P(\lambda) t(\lambda) a \Delta \lambda\left(\frac{D}{f}\right)^{2}
$$

$P(\lambda)$ is the flux number of solar photons (the factor 0.5 is atmospheric transmission), $t(\lambda)$ the overall system efficiency, $a$ the detector element area, and $\Delta \lambda$ the spectral element. The system efficiency is the total light loss in reflecting surfaces, transmission optics, beam splitters, monochromators, gratings, and the detectors quantum efficiency. The net through-put is the product of the contribution from the telescope and from the post focus instrument, i.e. $t(\lambda)=t_{t}(\lambda) t_{p f}(\lambda)$.

The number of reflecting surfaces in modern vacuum telescopes are $4-5$ (the Swedish telescope in La Palma has only three mirrors), and two transmission windows. Assuming that each reflecting surface is freshly coated with aluminum which gives mirror reflectivity 
will be $\simeq 0.88$ at $\lambda \simeq 5,500 \AA$, and window transmission 0.92 we get $t_{t}(5,500 \AA)=0.45$ to 0.51. The major light loss usually takes place in the focal plane instruments. For example, the estimates of Lites $(1987 a)$ for a LEST polarimeter give $t_{p f}(5,500 \AA) \leq 0.016$. The net efficiency will then be $t(5,500 \AA) \sim 0.008$. One may note that a major gain in the overall through-put of a solar telescope is likely to be achieved by clever design of focal plane instruments.

Assuming that photon noise dominates the signal to noise is given by $S=\sqrt{N}$. Let us assume that the angular subtense of the detector pixel elements $\Delta \Theta$ is about one-half of the angular resolution of the system and we get $\Delta \Theta \simeq 2 \sqrt{a} / f$. We may then express the relation between the telescope diameter $D$ and $S$ as (cf. MacQueen 1987):

$$
D \simeq \frac{2 S}{\Delta \Theta} \frac{1}{\sqrt{\pi 0.5 P(\lambda) t(\lambda) \Delta \lambda}}
$$

A signal to noise ratio $S \simeq 300$ will be appropriate for studies of spectral line profiles with $\Delta \lambda=0.010 \AA$. We take $P(5,500 \AA)=5.510^{13}\left[\mathrm{~cm}^{-2} A^{-1} \mathrm{~s}^{-1}\right]$ according to Lites $(1987 \mathrm{a})$, $\Delta \Theta=0.1$ arcsec and $t(\lambda) \simeq 0.008$ and find a requisite telescope diameter $D \sim 2.6 m$ for an integration time of $1 \mathrm{~s}$. We may conclude that solar (Stokes) polarimetric work is generally photon starved unless the telescope aperture exceeds 1-2 $\mathrm{m}$.

\subsection{Residual Polarization}

Observations and interpretations of spectral line profiles recorded in polarized light are invariably complicated by telescopic and instrumental polarization and the effects of yet unknown spatial averaging.

The stringent requirements on instrumental polarization in solar telescopes arises from their inability to provide high spatial resolution, - the polarimetry signal is blurred and therefore substantially weakened. Vector polarimetry will be more practical and simpler when the inherent spatial resolution is $\leq 0.3$ arcsec.

The aims of THEMIS and LEST are to reduce the parasitic polarization by using pointed telescopes with rotationally symmetric optics, at least before the position of the polarization analyzer. A recent study by McGuire and Chipman (1988) showed that even rotational symmetric optical systems give a net polarization effect. The effect depends largely on the f-ratio and the reflective coatings of the primary mirrors. In the case of LEST the upper bound of this polarization is $.68 \%$. However, since the effect is constant for a given telescope it can in essence be calculated and corrected (see Stenflo 1988; in McGuire and Chipman).

\section{Solar Seeing and Sites}

\subsection{Atmospheric optics}

Francois Roddier $(1981,1987)$ describes the effects of atmospheric turbulence on optical wavefront propagation. The incoming wavefront varies in amplitude $A(x)$ and phase $\Phi(x)$ across the entrance pupil. The image degradation arising from uncorrected amplitude variations ("scintillation") remains small. Image quality is considerably improved by correcting the phase error only. 
The variability of wavefront distortion can be represented by a characteristic spatial length (Fried parameter $r_{o}$ ), a disturbance lifetime (atmospheric de-correlation times $\tau_{o}$ ), and the angular isoplanatic angel $\Omega$. The Fried parameter (Fried 1966) can be expressed by

$$
r_{o}=0.185 \lambda^{6 / 5} \cos z^{3 / 5}\left[\int_{o}^{\infty} C_{n}^{2}(h) d h\right]^{-3 / 5}
$$

$\lambda$ is the wavelength, $z$ the zenith distance, and $C_{n}^{2}(h)$ is the structure function of the refractive index with height in the atmosphere.

The de-correlation time is due to refractive index inhomogeneities driven by wind across the optical beam and is of the order of

$$
\tau_{o} \simeq r_{o} / v
$$

where $v$ is the average wind velocity.

The angle $\Omega$ over which the wavefront perturbation remains approximately the same can be estimated from

$$
\Omega \simeq \frac{2}{3} r_{o} / h
$$

where $h$ is an average distance of the turbulent layers. Assuming $r_{o}=20 \mathrm{~cm}$ at $\lambda=$ $5,000 \AA$, and $v \simeq 10 \mathrm{~m} \mathrm{~s}^{-1}$ gives $\tau_{o} \simeq 20 \mathrm{~ms}$. For $h=4,000 \mathrm{~m}$ the isoplanatic angle becomes 7 arcsec.

It is essential to be aware of the inherent wavelength dependence of all three parameters, i.e. they all vary as $\lambda^{6 / 5}$. The effect of this is that the seeing becomes rapidly better with increasing wavelength.

\subsection{Meteorological Conditions of Solar Sites}

In the past decades both test measurements and actual astronomical observations have shown that high level island and coastal sites in certain latitude belts around the earth show superior performance to inland sites as far as night-time seeing is concerned (Walker 1984). The extensive JOSO testing campaigns performed in the Mediterranean, at the Western coast of the Atlantic ocean and on the Canary Islands (Brandt and Wöhl 1982; Brandt and Righini 1985a) have added some evidence to the validity of this general statement also for solar observations. The location of such good sites is closely connected to the large-scale global circulation pattern, with ascending motion near the equator and descending air masses in subtropical latitudes, forming the so-called trade wind system. High level sites in these latitudes are generally located in semi-permanent high pressure systems above an inversion layer, and are immersed in subsiding, dry and stable air masses (McInnes et al. 1974; Erasmus 1988).

Both the Hawaiian and the Canary Island archipelagos fulfill these conditions and their excellent suitability for astronomical observations is demonstrated by the fact that about half a dozen telescopes had been built and are being operated successfully on each of them. However, their actual performance, especially during daytime, depends critically on the 
microthermal conditions in the boundary layer ( 0 to $300 m$ above the sites), which are strongly influenced by the topography of the sites, heating of the ground, slope winds etc..

The Canary Islands are situated at a latitude of approx. $28 \mathrm{~N}$, longitude $17 \mathrm{~W}$ in the Azores high pressure system between 350 and $450 \mathrm{~km}$ from the African main land. The general climatological situation is well comparable with the one at Hawaii, i.e. a trade wind system with an inversion layer at heights between 1,200 and 1,600 $\mathrm{m}$ and subsiding stable air masses above these heights (Brandt and Righini 1985a,b).

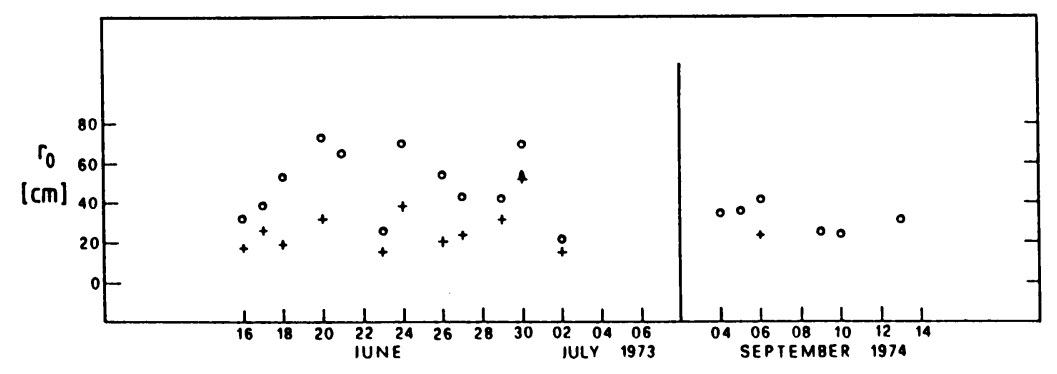

Figure 1: The Fried parameter $r_{o}$ deduced from radiosonde measurements. Circles include measurements up to $10 \mathrm{~km}$ height and crosses up to $17 \mathrm{~km}$. Barletti et al. 1977)

Early in-situ measurements of the temperature fluctuations with radio-sondes could be converted to values of $r_{o}$. The Figure 1 from Barletti et al. (1977) shows that most often $r_{o}$ $=20-30 \mathrm{~cm}$, and that occasionally $r_{o}$ exceeds $50 \mathrm{~cm}$. Other measurements have resulted in somewhat smaller values of $r_{o}$. Measurements with the $40 \mathrm{~cm}$ Newton vacuum telescope at Izaña in 1979 showed that $23 \%$ of the hourly measurements taken on 160 observing days yielded an instantaneous image sharpness of $\leq 1.2 \operatorname{arcsec}$ FWHM (cf. Brandt and Wöhl 1982). From a 7 day series of solar limb motion measurements in $1986 / 87$ by Kusoffsky (1988) shows a median $r_{o}$ of $12.4 \mathrm{~cm}$ with $10 \%$ of all values above $25 \mathrm{~cm}$. Moreover, a number of time sequences of granular evolution and narrow band filtergrams of sub-arcsec resolution have been observed with solar telescopes in the Canary Islands (Brandt et al. 1988; Scharmer 1989a; Title et al. 1989; Soltau 1989) thus proving the suitability for subarcsec solar observations. Programs that permit short exposure observations (less than $1 s)$ are usually getting diffraction limited images $\left(\sim \frac{1}{4}\right.$ arcsec $)$. One believes that the conditions at these sites may often be good for seeing better than $\frac{1}{4}$ arcsec.

\section{Image Sharpening Techniques}

\subsection{Reduction of Telescope Seeing}

Turbulence close to the telescope aperture degrades the seeing. Metalic surfaces and other local surfaces heated by the Sun will generate thermal convection and turbulence. A proturbing telescope in the wind is a turbulence generator. The latter is largely eliminated if the air can flow smoothly over the structure such as for the domeless and aerodynamic 
towers of the vacuum telescope of NSO/Sac Peak (Dunn 1969), the Japanese Hida telescope (Nakai 1980), and the Swedish telescope at La Palma (Wyller and Scharmer 1985, Scharmer 1989a). Thermal "trouble spots" may be identified by the aid of an IR camera and be eliminated by means of white paint or cover. Cooling panels works well to suppress local heating (Nakai and Hattori 1985, Pierce 1987).

Wind shake of exposed structures of heliostats and coelostats often gives rise to disturbing image motions. A Hammerschlag-Zwaan type windscreen has been mounted around the heliostat mirror of the McMath solar telescope and the windshake has been reduced substantially at minimum cost (Pierce 1989).

Evacuating the telescope light path eliminates internal seeing. Most modern solar telescopes are therefore vacuum telescopes (Dunn 1969, 1972; Mayfield et al. 1969; Zirin 1969; Livingston et al. 1976; Nakai and Hattori 1985; Wyller and Scharmer 1985; Soltau 1989). The major drawback of such systems is that thermal and mechanical stresses in the entrance windows give rise to optical aberration and polarization (Dunn 1984). The diameter/thickness ratio of a vacuum window is $\geq 10$, and vacuum telescope apertures are therefore in practize limited to less than $1 \mathrm{~m}$. Notable interest is invested in the possible use of helium gas in the telescope light path which may allow the use of a thin $(1-3 \mathrm{~cm})$ and larger diameter $(\geq 1 \mathrm{~m})$ entrance window. The idea of filling a telescope with helium was first put forward by B. Lyot and J. Rösch (Rösch 1965) and later tested by filling the Kitt Peak vacuum telescope with helium with promising result (Engvold et al. 1983). The advantages in using helium stem from its low refractive index, high thermal conductivity and relatively high viscosity. Detailed studies are presently carried out for the LEST project using a full scale mock-up steel tank of the telescope (Engvold et al. 1989). The microthermal fluctuations are quenched by filtering and circulating the gas.

\subsection{Correlation Trackers}

Image motion originates from instrument shake and guiding errors as well as from random wavefront tilts averaged over the telescope aperture due to atmospheric turbulence. Correction of wavefront tilt increases the spatial resolution by about a factor of two beyond the time averaged value, and the use of image stabilizers become rewarding.

In order to compensate for the image jitter caused by atmospheric seeing the system must be rather fast having a bandwidth of $\sim 10^{3} \mathrm{~Hz}$. This requires a fast and necessarily small active element in the optical path (cf. von der Lühe 1988b). The error signal that controls the active mirror is derived from measurements of the image motions via methods of pattern recognitions. For all trackers the tracking area must be $\leq \Omega^{2}$.

Pattern recognitions can be based on calculation of cross covariance (cross correlation) function of two images:

$$
C C(x, y)=\Sigma_{u} \Sigma_{v} f(x, y) * g(x+u, y+v)
$$

An alternative approach is to calculate the absolute difference of the images (Karud 1989).

$$
D(x, y)=\Sigma_{u} \Sigma_{v}|f(x, y)-g(x+u, y+v)|
$$

The $C C(x, y)$ function gives weight to each point in proportion to its brightness, which can lead to false, however small, image shifts. Low frequency intensity variations of the 
images must for this reason be removed before the cross correlation function is calculated.

Only areas of non-uniform intensity (intensity gradients) contain information about image displacement. Pixels from uniform areas are "dead" in terms of registering image shifts. Therefore, both algoritms can be optimized by multiplication each cross product, or difference, with a weight factor $w(x, y)$ given by the local intensity contrast. Oskar von der Lühe and collaborators (von der Lühe et al. 1989) have built a system for stabilization of image motion that utilizes the contrast of photospheric granulation. The tracker calculates the cross covariance function of a $16 \times 16$ pixel image by taking the Fourier transform of the two images, multiply one by the complex conjugate of the other, and take the inverse transform. This method is computationally expensive. The tracker developed by the Lockheed group to be used with the SOUP instrument and the OSL coordinated instrument package (Title 1989) uses a fast and simple third scheme that also utilizes the intensity gradients, but which can be used for small shifts ( $\sim 1$ pixel width) and conrasty images. Another simple approach has been developed for the THEMIS telescope using two crossed, one-dimensional resolving detectors. The error signal is derived from cross correlations of successive scans of these two detectors. The system is sensitive to image motions perpendicular to the scanning direction of the detectors and may thus loose track.

Stabilization of image motion i.⿲proves the overall image quality (Strehl Ratio) by a factor of 2 and is therefore highly rewarding.

The cross correlation technique is also successfully applied for measurements of horizontal flows in the photosphere (November 1986; Darvann 1988; Brandt et al. 1988; Simon et al. 1988) and in solar prominences (Darvann and Zirker 1989).

\subsection{Adaptive Optics}

Adaptive Optics (AO) is the technique in which the optics in a telescope are adjusted continuously with the aim of improving image quality for both short and long exposures. Adaptive optics in this broad definition corrects for telescope aberrations, tracking errors and dome seeing, as well as for atmospheric seeing outside the telescope dome (Beckers 1987). The term active optics is adopted for relatively slow adjustments to the telescope optics, aimed primarily at the correction of telescope aberrations.

An AO system consists of (i) a Wavefront Sensor (WFS), (ii) a complex servo loop which applies the error signal to (iii) the (iii) Adaptive Mirror (AM). A concept of an AO system is shown in Figure 2. A number of recent papers have reviewed the techniques of adaptive optics (Title 1985; Beckers 1987, 1989a; Dunn 1987; Hardy 1987; Merkle 1987).

Only the AO system of Lockheed has so far been in use in a solar telescope (Acton 1988). The adaptive mirror of the system consists of 19 hexagonal segments controlled by 57 piezoelectric driven actuators. The actuators have a travel of $5 \mu m$, corresponding to $\sim 10 \lambda$ in the visible, when operated at 40 volts. The Hartman type WFS operates on sunspots and pores. A separate "agile" flat mirror in the system is used to take out the overall wavefront tilt before the beam hits the adaptive, segmented mirror. The Lockheed system was run at the Tower Vacuum Telescope of NSO/Sacramento Peak in July 1988, and achieved occasionally near-diffraction limited images during average seeing conditions. It will be operated at this telescope during the next 2-3 years.

Any reasonable correction of the wavefront will lead to a point spread function con- 
sisting of an Airy disc superposed on a broad "halo" (Section 2.2). Current runs and simulations have shown that the residual of light in the "halo" is large when $r_{o}$ is larger than the mirror segments of the correcting system.

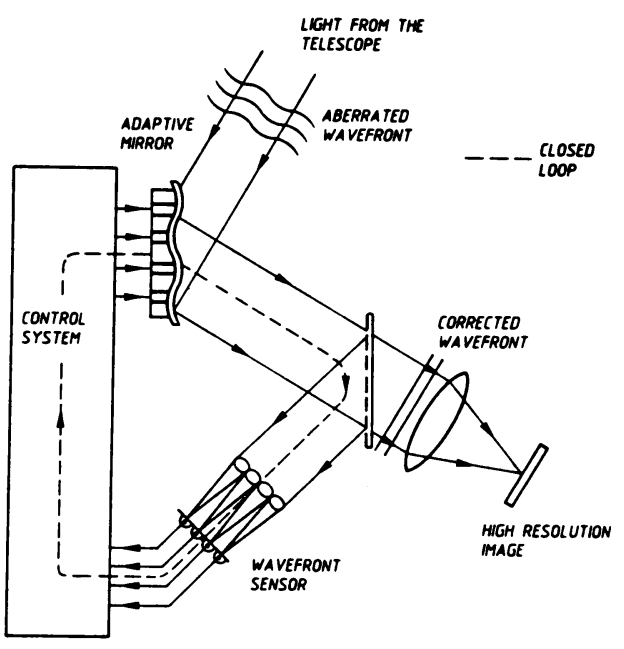

Figure 2: Principle of the application of adaptive optics in an astronomical telescope (Merkle 1987).

There is still a long way from the current successful demonstration of the Lockheed system to a general "user friendly" adaptive optics system for solar observations, but all fundamental and major technical problems appear to be solved. Given appropriate funding a routine-use $A O$ system will be available within a few years.

\subsection{Frame Selection}

In the cases of "broad" band $(\Delta \lambda \geq 1 \AA)$ observations one may continuously make short exposures and keep only the sharpest ones. Under the assumption that the atmospheric turbulence obeys the Kolmogorov distribution it is possible to quantify the improvements in spatial resolution from image selection. The degree of improvement is partly a function of the ratio $D / r_{o}$. Table 1 from Hecquet and Coupinot (1985) gives the improvement over the long exposure by selection of the listed percentile of best images.

From the Table 1 one finds that even a moderate image selection will result in a notable improvement in image quality. Beckers (1989b) stresses that the formula used for Table 1 applies only when the exposure time is short enough to "freeze" the seeing, and when $D$ represents the aperture diameter corresponding to the actual resolution of the telescope. The reason one hardly ever encounters a factor of four improvement in resolution from frame selection as predicted by Table 1 is that the telescope resolution is usually less than its theoretical limit. 
Table 1

$\begin{array}{ccccc}D / r_{o} & 76 \% \text { tile } & 10 \% \text { tile } & 1 \% \text { tile } & 0.1 \% \text { tile } \\ 3 & & & & \\ 5 & 2.0 & 2.7 & 2.9 & 3.0 \\ 10 & 1.9 & 3.1 & 3.6 & 4.0 \\ 20 & 1.5 & 2.4 & 3.0 & 3.8 \\ & 1.3 & 1.9 & 2.3 & 3.0\end{array}$

Values of $r_{o} \geq 10 \mathrm{~cm}$ are not uncommon at sites such as in the Canary Islands. Frame selection has been successfully applied to observations of photospheric granulation with the $50 \mathrm{~cm}$ Swedish solar telescope (Scharmer 1989a). This system "freezes" one image every $0.02 \mathrm{~s}$ and keeps the best frame for every $10 \mathrm{~s}$ period, corresponding to an image from the best 0.2 percentile. Providing $r_{o} \sim 15 \mathrm{~cm}$ one gets $D / r_{o} \sim 3.3$ and one expects to find diffraction limited images already in the 1 percentile. In the case of future large telescopes like $\operatorname{LEST}(D=2.4 \mathrm{~m})$ one would need $D / r_{o} \sim 8\left(r_{o} \sim 30 \mathrm{~cm}\right.$ at $\left.\lambda=6,000 \AA\right)$ in order to have 0.10 arcsec resolution images in the 0.1 percentile. At $\lambda=1.6 \mu m$ the same seeing will correspond to $D / r_{o} \sim 2.5$ and the diffraction limited images are in the 10 percentile.

\section{Instrument Development}

\subsection{Polarimeters}

Accurate polarimetry is best achieved through electro optical modulation of the light beam, thereby avoiding the use of moving parts (which inevitably produce noise) as well as eliminating any spurious polarization caused by seeing effects (since the modulation frequency can easily be made much larger than the seeing frequencies). The most simple and stable modulators with the best optical properties are the piezo elastic ones (PEMs), which give a sinusoidal modulation of the retardation at a frequency of typically $50 \mathrm{kHz}$ (Kemp 1969; Stenflo 1984a,b).

In order to overcome the much slower fram rates of multichannel detector arrays like CCD's compared to high frequency PEM's, one can introduce an optical demodulation scheme in the form of an electro optical "light chopper" in front of the detector, locked in frequency and phase to the polarization modulation (Stenflo and Povel 1985a,b). Although this optical demodulation scheme provides a solution to the PEM - CCD compatibility problem, it is rather complex and cumbersome to use. A far more elegant solution has been found and is under development by Povel and Stenflo (private communication). With this method the demodulation is done within the CCD itself, by shifting around the charges in synchrony with the polarization modulation. Only certain types of CCDs allow the charges to shifted at the rates required $(50-100 \mathrm{kHz})$, and the charges are only shifted by one column in the detector array at such rates. It is therefore necessary to interlace the exposed and unexposed (storage) areas, by optically blocking the light to every second of the array columns, which are used for buffer storage to allow a second image plane to exist. This is illustrated in Figure 3. The charges in the CCD are shifted back and forth between the odd and even columns in synchrony with the PEM modulation. 
A prototype of the LEST polarimetry system, including the optical modulation package with two PEMs (which will sit in the LEST secondary focus) and the CCD demodulation scheme (based on synchronous shifting of the charges in the CCD) is being developed for LEST in Zürich and will be tested out in a complete observing system at the Astrophysical Observatory Arosa.

Figure 3: Schematics of the synchronous shift of charges of the CCD detector array of the ETH polarimeter.

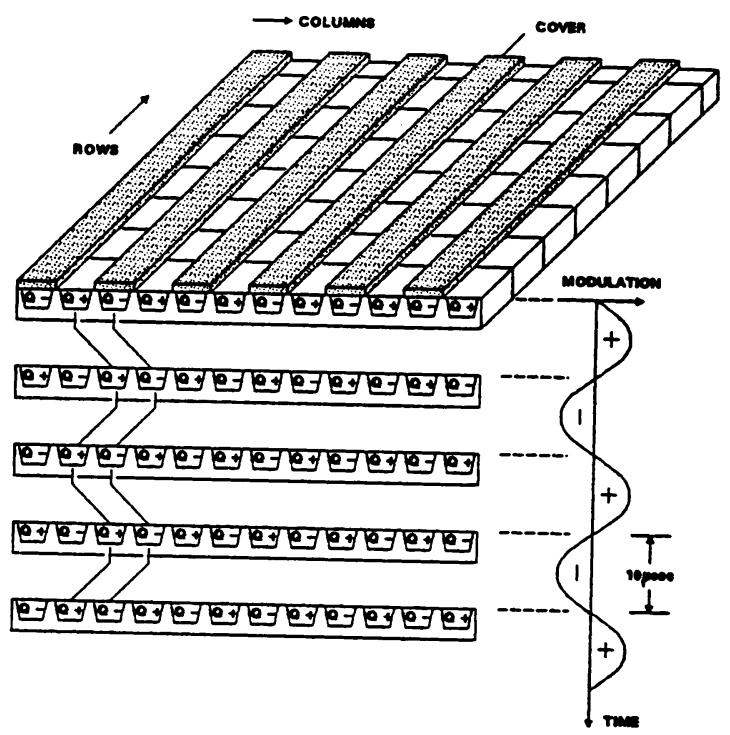

Two new polarimeter systems are presently under development in the USA, one at the High Altitude Observatory (HAO) in Boulder, and the other at the Institute for Astronomy, University of Hawaii (UH). In both systems the polarization modulation is performed by the mechanical rotation of a retardation plate (Lites 1987b). Both systems also use CCD arrays for 2-D imaging. The images of the four Stokes parameters are formed through linear combination of consequtive images read out at different position angle setting of the rotating retarder.

The HAO system, called the Advanced Stokes Polarimeter, will be set up at the NSO Tower Vacuum Telescope at Sacramento Peak. It will use a spectrometer with moderately high spectral and 1-D spatial resolution, emphasizing high-quality line profile informations for quantitative fluxtube analyzis and Stokes inversion techniques.

The UH system on the other hand, called the Imaging Vector Magnetograph, uses a tunable narrow-band filter, thereby sacrifying spectral informations to obtain fast 2-D spatial imaging of the Stokes parameters, with the aim of relating the changing morphology and vector magnetic fields to solar flares.

\subsection{Narrowband Filters and Spectrographs}

A Universal Birefringent Filter (UBF) in tandem with a Fabry-Perot interferometer is being developed for 2-D spectroscopy by Bonaccini et al. (1989). The filter combination 
can be operated in the range $\lambda \lambda 4,000-7,000 \AA$ with a spectral bandwidth of $\sim 20 \mathrm{~m} \AA$. The net peak transmission is $4 \%-12 \%$, and the positioning accuracy is $1 \mathrm{~m} \AA$. A Triple-FabryPerot Universal Filter is installed and tested on the $30 \mathrm{~cm}$ refractor of the CSIRO Solar Observatory (Bray 1988). Various options of multichannel universal filters are considered by $\mathrm{Ai}$ and $\mathrm{Hu}(1985)$ and collaborators.

A versatile Echelle grating spectrograph is being designed and planned for THEMIS and LEST (Mein 1989). The design includes the use of one large mirror ion Ebert-Fastie mounting instead of the more commonly used two midle-sized collimator and camera mirrors.

\section{New Telescope Facilities in the Canary Islands}

\subsection{The German Solar Telescope Facilities at Izaña}

The German solar telescope installations at Observatorio del Teide, Izaña, consist of the $70 \mathrm{~cm} \mathrm{f} / 66$ Vacuum Tower Telescope (VTT) of Kiepenheuer-Institut, Freiburg, the 45 $\mathrm{cm}$ f/56 Gregory-Coudé of Göttingen Observatory, Göttingen, and the smaller GermanSpanish $40 \mathrm{~cm}$ Newton telescope (Schröter et al. 1985).

The VTT has a classic coelostat configuration and a vertical tower telescope (Mehltretter 1975). The vacuum window is located underneath the coelostat and the window cell has provisions for cooling. The dome is a removable jaw-type similar to the dome of the $60 \mathrm{~cm}$ vacuum telescope of NSO/Kitt Peak (Livingston et al. 1976). The telescope had "first light" in 1987, and the telescope has occasionally approached diffraction limited performance during 1988 (Soltau 1989). The VTT will be equipped with a Multichannel Subtraction Double Pass spectrograph of Meudon (Mein 1977) for 2-D spectroscopic studies. Also the Italian stable UBF will be installed in this telescope.

The Gregory-Coudé telescope was moved from the former German station at Locarno and has been in operation at Izaña since July 1986 (Kneer and Wiehr 1989). The spectrograph is a Czerny-Turner type with collimator and camera mirror focal lenghts $10 \mathrm{~m}$. A system for speckle imaging is being prepared.

The spectroscopic studies of the Göttingen group are focused on small scale dynamic of quiescent and active regions, sunspots and prominences.

\subsection{The Swedish Solar Station in La Palma}

\subsubsection{Instrumentation}

The Swedish solar telescope at Roque de los Muchachos, La Palma (Scharmer et al. 1986) is patterned after the vacuum tower telescope of NSO/Sac Peak (Dunn 1964, 1985). The combination of an optically simple and good system, and a superb site has made this telescope one of the very best in the World for high resolution studies of the Sun.

The domeless turret design is very compact and its aerodynamic shape gives a minimum of local disturbance and eliminates dome seeing completely. Furthermore, the mirrors are located inside vacuum thus eliminating convection near heated surfaces. The $50 \mathrm{~cm}$ doublet achromatic lens that serves as vacuum window to the telescope. The lens is made from BK7 and F2 glasses, and the focal length is $22.35 \mathrm{~m}$. The lens cell is designed to 
avoid any heating of the lens by the cell. Thermal gradients usually appears in vacuum windows due to local heating which lead to focus changes in the telescope and to spherical aberration (Dunn 1972; Mehltretter 1979). Optical tests have shown that the Swedish telescope has virtually diffraction limited performance (Scharmer 1989a).

The real-time image acquisition and selection system is a valuable asset of the observatory. An important property of the system is that it allows for simultaneous recordings with two synchronized CCD cameras. The two images, which can be a spectrum and its corresponding slit-jaw picture or images at two different wavelengths, "freeze" the atmospheric seeing motion (exposure times $1 / 30$ s or $1 / 60$ s) and provide unambiguous identifications of corresponding structures. The real-time image selector "grabs" and stores only the best images in a selectable time interval which is commonly set to be $10 \mathrm{~s}$. The system has been operated successfully to obtain extended time series of photospheric granulation (Scharmer 1989a). It is expected to work for chromospheric structures and dark filaments as well.

\subsubsection{Recent Results}

The best time sequence of photospheric granulation that has been analyzed so far covers 79 minutes and was obtained June 16, 1987 (Brandt et al. 1988). The spatial resolution was $\approx \frac{1}{4}$ arcsec over 18 arcsec frame size. Local flow velocities were found on the average to be about $1.6 \mathrm{krn} \mathrm{s}^{-1}$. These speeds were larger than similar data recorded earlier with lower spatial resolution (Title et al. 1989). The most spectacular discovery of this study was that of a vortex of $5,000 \mathrm{~km}$ in diameter and which persisted for the duration of the sequence. Similar recent studies by Müller (1989b) did not find vortex structures.

Figure 4: Image of large sunspot observed July 26, 1988, with the Swedish vacuum solar telescope at La Palma, Canary Islands. The observations were made through a $25 \AA$ wide filter centred on $4686 \AA$. The spatial resolution is about $\frac{1}{4}$ arcsec. The image scale is $18 \times 25$ arcsec $^{2}$. (Courtesy G.B. Scharmer)

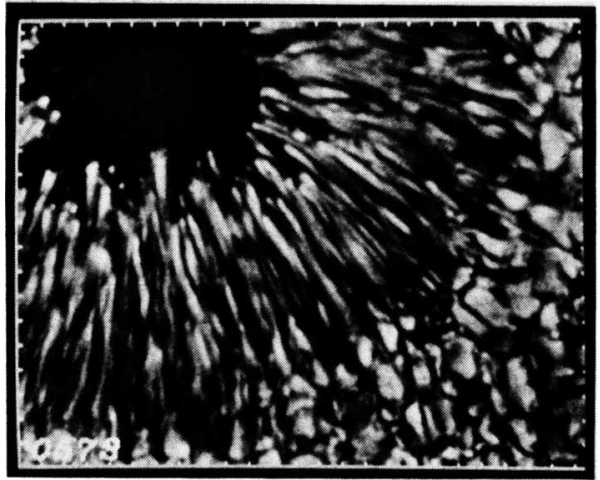

A new time series of a medium size sunspot reveals a stunning wealth of dynamic structures (Scharmer 1989b). Figure 4 shows a $18 \times 25 \operatorname{arcsec}^{2}$ view of the penumbra and near by photosphere from this time series. A study of Darvann and Kusoffsky (1989) using a 19 minutes long series of photosperic granulation found that granulation lifetimes decreased from about 12 minutes close to the sunspot pore region to 5 minutes away from the pore. 


\section{Near Future Solar Telescope Projects}

\subsection{THEMIS}

The French polarization-free solar telescope "Télescope Héliographique pour l'Etude du Magnetism et des Instabilités Solaires" - (THEMIS) will be built at Izaña, on the island of Tenerife, Canary Islands. The site is being prepared for construction of the THEMIS building, and the first light in the instrument is expected in 1991.

The pointing Ritchey-Chretien telescope system of $90 \mathrm{~cm}$ aperture will be evacuated and sealed by two windows (Mein and Rayrole 1985, 1988). The prime focus (f/17) is the location of the polarization modulator. A high precision correlation tracker and a "tip-tilt" (active) mirror between the prime and secondary foci will be used to stabilize the residual jitter of the solar image from seeing and telescope vibrations. The main instrument, the Multichannel Subtractive Double Pass (MSDP) Echelle spectrograph (Mein 1977, Mein and Rayrole 1988) is mounted behind the secondary focus (f/60).

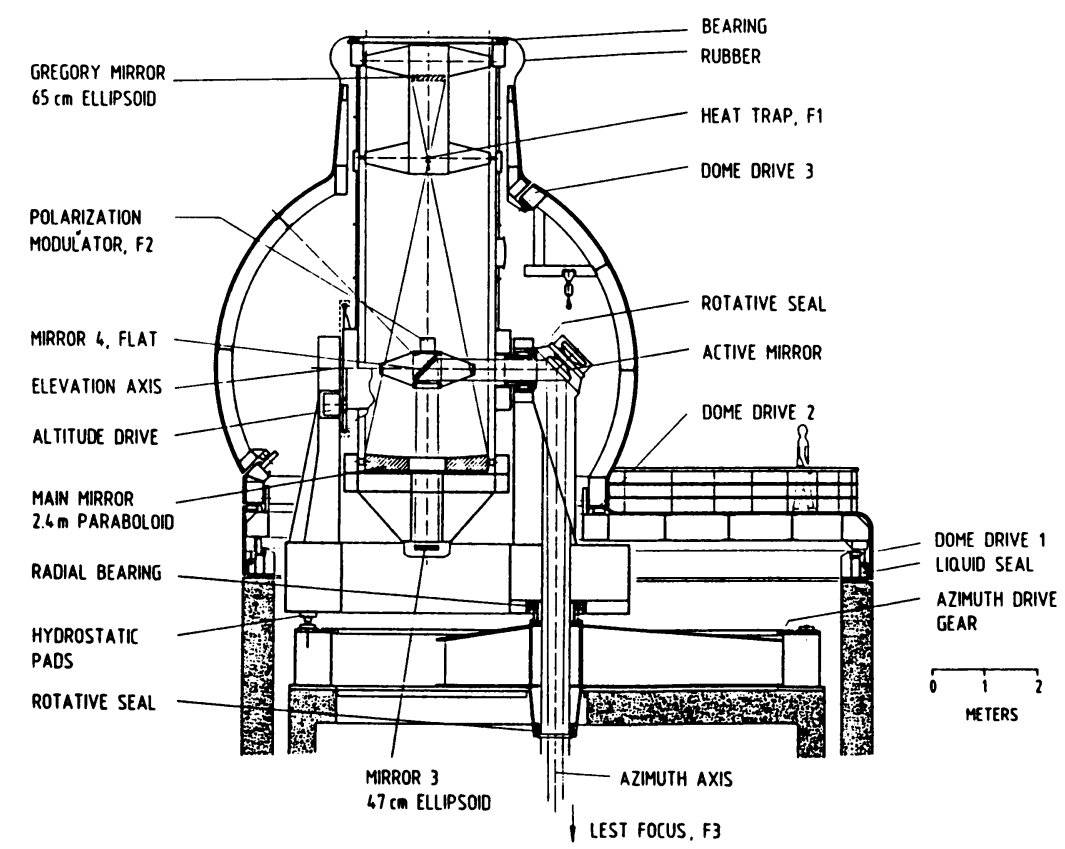

Figure 5: Vertical cross section of the top part of the LEST (Andersen et al. 1985).

\subsection{Large Earth-based Solar Telescope - LEST}

LEST is the most ambitious ground based solar telescope program today. The current design is shown in Figure 5 (Andersen et al. 1984). The LEST aperture is $2.4 m$ and the diffraction limit of the modified Gregorian system is 0.05 arcsec at $\lambda 5,000 \AA$. Evacuation becomes impractical for such a large telescope since it will require a rather thick entrance 
window. It is instead suggested to fill the internal light path with helium gas at nearly ambient air pressure to make possible to use a thin entrance window.

The LEST will be placed either on La Palma, Canary Islands (2 $360 \mathrm{~m}$ a.s.l.) or on the cinder cone $P u$ ' $u$ Poli'ahu at Mauna Kea, Hawaii (4 $150 \mathrm{~m}$ a.s.l.). Besides utilizing a very good site for daytime observations LEST will seek to achieve near diffraction limited resolution within a small field of view by means of image sharpening techniques mentioned above.

The multi-national, non-profit organization behind the project, the LEST Foundation, presently counts 10 member countries (Stenflo 1985; Wyller 1986). The construction of LEST could start in 1992 and the telescope may be ready for first light in 1995.

\subsection{Solar Missions in Space}

The Solar and Heliospheric Observatory - SOHO is a space mission for studies of the solar interior and the outer solar atmosphere. The payload shall include six instruments for studies of structures and dynamics of the Sun's chromosphere and corona (Domingo and Poland 1989).

Two of the SOHO instruments, Solar Ultraviolet Emitted Radiation (SUMER) and Coronal Diagnostic Spectrometer (CDS), will both build up 2-D images in the order of seconds by scanning their spectroscopic slits across the solar disk with $\sim 2$ arcsec resolution. A third instrument, the Extreme-ultraviolet Imaging Telescope (EIT) provides high resolution images of the whole Sun at several temperatures. Telemetry of the data will take place via NASA's Deep-Space Network during four designated periods per day.

SOHO is currently scheduled for launch in July 1995 and is being designed for a lifetime of two years, but it will be equipped with sufficient on board consumables for an extra four years.

The Orbiting Solar Laboratory (OSL) of NASA is a scaled down version of the former Solar Optical Telescope (SOT) and the High Resolution Solar Observatory (HRSO). OSL will be a free flying, polar orbiting instrument for high spatial and temporal resolution of the Sun over a spectral range from the X-ray to the near IR (Title 1989). The OSL satellite will harbour a $1 \mathrm{~m}$ aperture telescope optimized for $\lambda \lambda 2,000-11,000 \AA$, which feeds a narrow-band filter, a set of fixed broad-band filters, and a visible light Echelle spectrograph.These three instruments are mounted together in a common structure; the Coordinated Instrument Package (CIP). The CIP will have 0.13 arcsec resolution over a 3.9 arcmin field-of-view. An ultraviolet spectrograph and an XUV/X-ray imager are planned co-pointing and co-aligned instruments with overlapping fields of view. The latter is probably an array of normal incidence telescopes with multi layer coatings. The UV instrument will be Naval Research Laboratory's High Resolution Telescope and Spectrograph (HRTS), which covers the wavelength range $\lambda \lambda 1,175-1,700 \AA$ that contains numerous bright emission lines for studies of the chromosphere and chromosphere-corona transition region. The spatial resolution of HRTS is $\sim 1$ arcsec. The planned launch dates of OSL are 1995-96, and the design lifetime will be 3 years even though it is reasonable to expect that a full scale operation could be technically possible for $8-10$ years.

The HRTS instrument will also be flown on rockets during the next few years, presumably as often as about one per year. 


\section{Prospects for High Resolution Observations of the Sun}

New optical telescopes in space (SOHO and possibly OSL) and ground-based (the German and Swedish installations in the Canary Islands, and the future THEMIS and LEST) challenge existing high resolution solar telescopes like Pic-du-Midi (Müller 1989), NSO/Sac Peak (Dunn 1969), Big Bear Solar Observatory (Zirin 1969), and Hida (Nakai and Hattori 1985). The advance of techniques for high resolution imaging and the utilization of excellent sites for daytime seeing will make angular resolution beyond 0.2 arcsec in solar observations within reach in a few years.

\section{Acknowledgement}

Informations on polarimeters were kindly provided by Jan Olof Stenflo.

\section{References}

Acton, D.S.: 1988 "High Spatial Resolution Solar Observations", Sac Peak Workshop Aug. 22-26 (to be published)

Ai Guoxiang and Hu Yuefeng,: 1985 LEST Technical Report No. 14

Andersen, T.E., Dunn, R.B., and Engvold, O.: 1985 LEST Technical Report No. 7

Barletti, R., Ceppatelli, G., Paternò, L., Righini, A., and Speroni, N.: 1977 Astron. Astrophys. 54, 649

Beckers, J.M.: 1986 SPIE Proceedings 628, 190

Beckers, J.M.: 1987, LEST Technical Report No. 28, p.55

Beckers, J.M.: 1989a "Solar and Stellar Granulation", Eds.: R.J. Rutten and G. Severino, NATO ASI Series, Kluwer Academic Publishers, p.43

Beckers, J.M.: 1989b ibid p.55

Bonaccini, D., Cavalini, F., Ceppatelli, G., and Righini, A.: 1989 Astron. Astrophys. (in press)

Brandt, P.N. and Wöhl, H.: 1982, Astron. Astrophys. 109, 77

Brandt, P.N. and Righini, A.: 1985a, LEST Technical Report No. 11

Brandt, P.N. and Righini, A.: 1985b, Vistas in Astronomy 28, 437

Brandt, P.N. Scharmer, G.B., Ferguson, S., Shine, R.A., Tarbell, T.D. and Title, A.M.: 1988, Nature 335, 238

Bray, R.J.: 1988 LEST Technical Report No. 35

Darvann, T.A.: 1988 "High Spatial Resolution Solar Observations" 10th Sac Peak Summer Workshop Aug. 22-26 (Ed.: O. von der Lühe)

Darvann, T.A. and Kusoffsky, U.: 1989 "Solar and Stellar Granulation", Eds.: R.J. Rutten and G. Severino, NATO ASI Series, Kluwer Academic Publishers, p.313

Darvann, T.A, and Zirker, J.: 1989 IAU Meeting on Solar Prominences Hvar, Yugoslavia September 27-29.

Dere, K.: 1987 Solar Physics 114, 223 
Domingo, V. and Poland, A.I.: 1989 "THE SOHO MISSION", Scientific and Technical Aspects of the Instruments, ESA SP-1104, p.7

Dunn, R.B.: 1964 Appl. Optics 3, 1353

Dunn, R.B.: 1969 Sky \& Telescope 38, 368

Dunn, R.B.: 1972 Space Research XII, 1657

Dunn, R.B.: 1984 LEST Technical Report No. 3

Dunn, R.B.: 1985 Solar Physics 100, 1

Dunn, R.B.: 1987 LEST Technical Report No. 28, p.243

Engvold, O., Dunn, R.B., Livingston, W.C., and Smartt, R.: 1983 Applied Optics 22, 10

Engvold, O., Andersen, T.E., Carlsson, M., Jensen, J.R., and Klim, K.: 1985 LEST Technical Report No. 15

Engvold, O. et al. : 1989 (To be published)

Erasmus, D.A.: 1988, LEST Technical Report No. 31

Fried, D.L.: 1966, J. Opt. Soc. Am. 56, 1372

Hardy, J.W.: 1987 LEST Technical Report No. 28, p.137

Hecquet, J. and Coupinot, G.: 1985 J. Optics (Paris) 16, 21

Karud, J.O.: 1989 Master Thesis, Univ. Oslo. (to be published)

Kemp, J.C.: 1969, J. Opt. Soc. Am. 59, 950

Kneer, F. and Wiehr. E.: 1989 "Solar and Stellar Granulation", Eds.: R.J. Rutten and G. Severino, NATO ASI Series, Kluwer Academic Publishers, p.13

Knox, K. and Thompson, B.: 1974 Astrophys. J. Letters 193, L45

Kusoffsky, U.: 1988 (unpublished)

Lites, B.W.: 1987a, LEST Technical Report No. 22

Lites, B.W.: 1987b, LEST Technical Report No. 23

Livingston, W.C., Harvey, J., Pierce, A.K., Schrage, D., Gillespie, B., Simmons, J., and Slaughter, C.: 1976 Applied Optics 15, 33

MacQueen, R.M.: 1987 LEST Technical Report No. 24

Martin, R. and Hartley, K.: 1985 Vistas in Astronomy 28, 555

Mayfield, E., Vrabec, D., Rogers, E., Janssens, T., and Becker, R.: 1969 Sky \& Telescope 37, 208

McGuire, J.P. and Chipman, R.A.: 1988 LEST Technical Report No. 36

McInnes, B., Hartley, M. and Gough, T.T.: 1974, Observatory 94, 14

Mein, P.: 1977 Solar Phys. 54, 45

Mein, P.: 1989 LEST Technical Report No. 37

Mein, P. and Rayrole, J.: 1988 "High Spatial Resolution Solar Observations", 10th Sac Peak Summer Workshop Aug. 22-26 (Ed.: O. von der Lühe)

Mehltretter, J.P.: 1975 JOSO Annual Report p.

Mehltretter, J.P.: 1979 J. Optics 10, 93 
Merkle, F.: 1987, LEST Technical Report No. 28, p.117

Müller, R.: 1989a "Solar and Stellar Granulation", Eds.: R.J. Rutten and G. Severino, NATO ASI Series, Kluwer Academic Publishers, p.9

Müller, R.: 1989b (Private communication)

Nakai, Y.: 1980 Proceedings of The Japan-France Seminar on Solar Physics. Eds.: F.Moriyama, J.C.Henoux, 285

Nakai, Y. and Hattori, A.: 1985 Mem. Fac. Sci., Kyoto University, Ser. Physics, Astrophysics, Geophysics and Chemistry 36, No. 3, 385

November, L.: 1986 Applied Optics 25, 392

Pierce, A.-K.: 1987 Solar Physics 107, 397

Raffi, G.: 1988 "ESO Conference on Very large Telescope and Their Instrumentations". Garching 21-24 March 1988. Ed.:M.-H. Ulrich, p.1061

Roddier, F.: 1981 Progress in Optics 19, 333

Roddier, F.: 1987 LEST Technical Report No. 28, p.7

Roddier, F. and Roddier, C.: 1986 Proceedings SPIE 688, 298

Rösch, J.: 1965 Applied Optics 4, 1672

Scharmer, G.B.: 1989a "Solar and Stellar Granulation", Eds.: R.J. Rutten and G. Severino, NATO ASI Series, Kluwer Academic Publishers, p.161

Scharmer, G.B.:1989b (Private communication)

Schröter, E.H., Soltau, D., and Wiehr, E.: 1985 Vistas in Astronomy 28, 519

Simon, G.W., Title, A.; Topka, K., Tarbell, T., Shine, R., Ferguson, S., Zirin, H., and the SOUP Team: 1988 Astrophys. J. 327, 964

Soltau, D.: 1989 "Solar and Stellar Granulation", Eds.: R.J. Rutten and G. Severino, NATO ASI Series, Kluwer Academic Publishers, p.17

Stachnik, R.V., Nisenson, P., and Noyes, R.W.: 1983 Astrophys. J. 271, L37

Stenflo, J.O.: 1984a, Appl. Optics 23, 1267

Stenflo, J.O.: 1984b, LEST Foundation Technical Report No. 4

Stenflo, J.O.: 1985 Vistas in Astronomy 28, 571

Stenflo, J.O., Povel, H.: 1985a, Appl. Optics 24, 3893

Stenflo, J.O., Povel, H.: 1985b, LEST Foundation Technical Report No. 12

Title, A.M., and Tarbell, T.D. et al.: 1987 in "High Resolution Solar Physics II", NASA Conf. Publ. 2483, 55. Eds.: R.G. Athay and D. Spicer

Title, A.: 1985 "High Resolution in Solar Physics". Lecture Notes in Solar Physics No. 233, 51

Title, A.M.: 1989 "Solar and Stellar Granulation", Eds.: R.J. Rutten and G. Severino, NATO ASI Series, Kluwer Academic Publishers, p.29

Title, A.M. et al.: 1989, publication in preparation

Walker, M.F.: 1984, in "Site Testing for Future Large Telescopes", ESO Conf. and Workshop Proc. No. 18, ed. Ardeberg and Woltjer, 3 
von der Lühe, O.: 1988a JOSA A 5, 721

von der Lühe, O.: 1988b Astron. Astrophys. 205, 354

von der Lühe, O. and Zirker, J.B.: 1988 "High Resolution Imagery by Interferometry", NOAO/ESO Conf. 15-18 March, Garching, FRG (Ed.: F. Merkle)

von der Lühe, O., Widener, A.L., Rimmele, Th., Spence, G., Dunn, R.B., and Wiborg, P.: 1989 Astron. Astrophys. (submitted)

Wyller, A.A.: 1986 LEST Large Earth-based Solar Telescope - An Overview. LEST Foundation, Royal Swedish Academy of Sciences, Stockholm

Wyller, A.A., Scharmer, G.B.: 1985 Vistas in Astronomy 28, 467

Zirin, H.: 1969 Sky \& Telescope 51, 215 